Proc. XIX International School of Semiconducting Compounds, Jaszowiec 1990

\title{
IV-VI NARROW-GAP SUPERLATTICES
}

\author{
F. F. Sizov \\ Institute of Semiconductors of the Academy of Sciences of the Ukr. SSR, \\ Kiev-252 650, USSR \\ (Received August 8, 1990)
}

\begin{abstract}
Electronic structure, optical and electrical properties of IV-VI superlattices and multiple quantum wells (MQW) are discussed and compared with corresponding properties of IV-VI bulk semiconductors. In particular, the types of superlattice structure and MQW formed from various constituents are discussed.
\end{abstract}

PACS numbers: $68.65+g$

\section{Introduction}

The research on semiconductor superlattices (SL) was initiated in 1969 with the proposal by Esaki and Tsu [1] for a solid state system with an effective one-dimensional periodic potential which was to result from alternating ultrathin semiconducting layers. Two types of SL were envisioned: first, consisting of one semiconductor with the level and/or type of doping alternating along certain direction, and second - consisting of different semiconductors as constituents (Fig. 1). In both cases the SL are solid-state structures in which beside the lattice periodic potential there is a one-dimensional potential with the period $d$ essentially greater than the lattice constant $\left(a_{o}\right)$. Creation of similar one-dimensional potential in solids, however, realized with the use of acoustic waves, was firstly proposed by Keldysh as early as 1962 [2].

The presence of an additional SL periodic potential changes the electronic spectrum of a semiconductor. The Brillouin zone is divided into a serious of minizones giving rise to narrow subbands separated by minigaps. Thus the superlattices possess new properties that are not exhibited by homogeneous semiconductors. This fact opens a whole spectrum of possibilities of purposeful design of entirely new electronic devices (see, e.g. [3-5]).

The first IV-VI compositional SL $\left(\mathrm{PbTe} / \mathrm{Pb}_{1-x} \mathrm{Sn}_{x} \mathrm{Te}\right)$ were grown in Japan by hot wall epitaxy (HWE) on $\mathrm{BaF}_{2}$ substrates [6]. The first IV-VI doping SL 
(a)

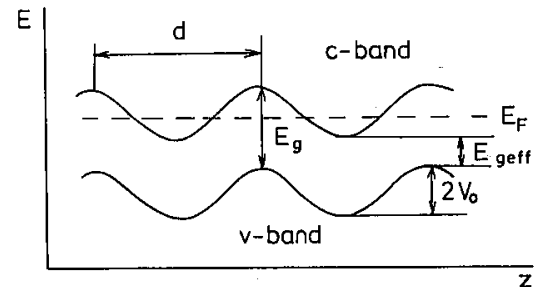

(b)

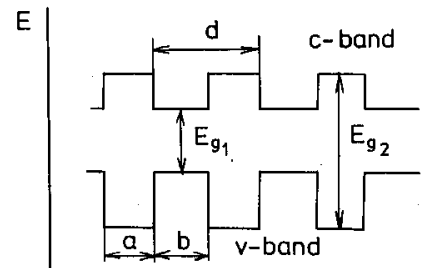

Fig. 1. Spatial variations of the band edges in (a) doping (nipi) superlattices and (b) compositional superlattices. Notation: $E_{g}$ - band gap, $E_{g e f f}$ - effective band gap, $2 V_{0}$ - nipi modulation potential, $E_{F}$ - Fermi level, z - space coordinate.

Fig. 2. (a) Structure of $\mathrm{Pb}_{1-x} \mathrm{Sn}_{x} \mathrm{Te} \mathrm{MQW}$ laser and (b) carrier concentration profile (after [18]).

(PbTe nipi structures) were grown by the same method in the same group [7]. A little bit later, $\mathrm{PbTe}$ nipi structures were obtained in Austria [8]. Approximately at the same time the strained, containing dislocations IV-VI SL (PbTe/PbSe), $(\mathrm{PbTe} / \mathrm{PbS}),(\mathrm{PbSe} / \mathrm{PbS})$ were obtained in the Soviet Union [9]. Later, the highest critical temperatures of superconductivity (as high as $T_{c} \approx 5.5 \mathrm{~K}$ ) in semiconductors were observed in these structures $[10,11]$. Growth of IV-VI/III-V ( $\mathrm{PbTe} / \mathrm{InSb}$ ) SL by laser assisted evaporation method was reported in [12].

Historically, systematic investigations on physics and growth techniques of IV-VI semiconductors began in the late fifties [13]. These semiconductors are mainly used for applications in IR optoelectronics as IR-detectors for wavelengths $\lambda \approx 2-15 \mu \mathrm{m}$ and as IR diode lasers for the region of wavelengths $\lambda \approx 3-45 \mu \mathrm{m}$. Sensitive photodiodes operating at $T=77 \mathrm{~K}$ at BLIP regime (BLIP - Background Limited Infrared Photodetector) are mainly manufactured from $\mathrm{PbTe}$ and $\mathrm{Pb}_{1-x} \mathrm{Sn}_{x}$ Te [14]. IV-VI laser diodes, as a rule, are used in spectroscopic applications. Depending on the wavelength of the emission $\lambda_{e}$, these devices can be used at temperatures $T \leq 80 \mathrm{~K}$ in CW operation (though higher temperatures of CW operation has been reported [15]). In pulsed operation mode they are used at $T \leq 120 \mathrm{~K}$. The rule is that the operation temperature must be lowered when the value of $\lambda_{e}$ is decreased. The goal of the design IV-VI SL and quantum well (QW) devices in applications in IR optoelectronics is to obtain more effective devices, operating at elevated temperatures. For instance, it was shown in [16] that it is 
possible to obtain $\mathrm{PbTe}$ doping superlattices operating at $T=77-90 \mathrm{~K}$ in the photoconductive regime with detectivities close to the theoretical limit. The use of $\mathrm{PbTe} \mathrm{QW}$ with lattice-matched $\mathrm{Pb}_{1-x} \mathrm{Eu}_{x} \mathrm{Se}_{y} \mathrm{Te}_{1-y}$ confinement layers enabled to elevate the temperature of $\mathrm{CW}$ laser operation up to $174 \mathrm{~K}\left(\lambda_{e}=4.39 \mu \mathrm{m}\right)$ and up to $T=270 \mathrm{~K}$ for pulsed operation $\left(\lambda_{e}=3.88 \mu \mathrm{m}\right)$ [17]. PbSnTe multiple QW (MQW) lasers were obtained in [18] and were shown to operate in pulsed mode up to $T=204 \mathrm{~K}\left(\lambda_{e}=6 \mu \mathrm{m}\right)$ or in $\mathrm{CW}$ mode up to $T=130 \mathrm{~K}\left(\lambda_{e}=6.6 \mu \mathrm{m}\right)$.

The possibility to raise the operating temperature of the IR SL or MQW optoelectronic devices arises because of several special features of their construction. For example, MQW lasers are very suitable for high-temperature $;$ and more effective operation since an inversion of the population can be easily rbtained by an appropriate doping sequence of the constituents (Fig. 2). By choosing another constituents or doping one can change the type of SL in such a way that electrons and holes will be spatially separated resulting in longer recombination times.

\section{General features of IV-VI semiconductors}

IV-VI compositional and doping SL show a number of different properties not found in such structures made of III-V or II-VI semiconductors. The novel features are due to some special properties of the bulk materials:

1. IV-VI semiconductors crystallize in the cubic $\mathrm{NaCl}$ structure.

2. These semiconductors have direct band gap at $\mathrm{L}$ point of the Brillouin zone which gives rise to the multivalley band structure.

3. The electron and hole masses at the conduction band and valence band edges are nearly equal (neglecting small corrections due to interactions with remote bands). The surfaces of constant energy are almost mirror-like ellipsoides of revolution with the $\langle\overline{1} 11\rangle$ direction as their main axes.

4. Free carriers in the conduction band are due to the chalcogen vacancies (each gives two electrons) and in the valence band - due to the metal vacancies (each gives two holes). The energy levels of these vacancies are deep in the bands. For this reason the freeze-out effect of carriers is absent in these materials.

5. Static dielectric permittivity is high $\left(\varepsilon_{0} \geq\left(10^{2}-10^{3}\right)\right)$ and strongly temperature dependent because of the softening of the transverse optic mode which is indicative of the proximity of the structural phase transition.

As a consequence of these features, for example, the Coulomb scattering is inefficient in limiting the carrier mobility at low temperatures. The temperature dependence of electron and hole mobility in the temperature range $T \approx 30-300 \mathrm{~K}$ is governed by long wave optic phonon and acoustic phonon scattering. Because of the symmetry of the cubic $\mathrm{NaCl}$ structure the optical absorption in the bulk IV-VI crystals does not depend on the polarization of light. In SL it may depend very 
strongly on the polarization as well as on the direction of the propagation with respect to SL axes, because the constant energy ellipsoids directions can be oblique to any SL axis $[19,20]$. Due to high values of $\varepsilon_{0}$ there are no shallow hydrogen-like bound states in the gap, there are only deep impurity states that can be both in the gap and in the conduction or valence bands. Because of the electrical activity of the vacancies, the conductivity type and the value of carrier concentrations in IV-VI constituents of a SL may, to a certain degree, be controlled by the growth temperature and the vapor pressure of the chalcogen.

Several SL systems were already realized by various growth methods from IV-VI semiconductors. Table I lists the IV-VI SL grown so far giving also the methods of growth and the types of SL obtained. The possible types of IV-VI SL are shown schematically in Fig. 3.

TABLE I

Types of IV-VI SL and MQW and the methods of their growth

\begin{tabular}{|c|c|c|c|}
\hline Materials & $\begin{array}{l}\text { Growth } \\
\text { method }\end{array}$ & $\begin{array}{l}\text { Type } \\
\text { of SL } \\
\end{array}$ & References \\
\hline $\mathrm{PbTe} / \mathrm{Pb}_{1-x} \mathrm{Sn}_{x} \mathrm{Te}$ & HWE & $\mathrm{I}$ & {$[21-26]$} \\
\hline$(x \leq 0.2)$ & HWE & I' & {$[27,28]$} \\
\hline \multirow{2}{*}{$\begin{array}{l}\mathrm{Pb}_{1-x} \mathrm{Sn}_{x} \mathrm{Te} / \mathrm{PbTe}_{1-y} \mathrm{Se}_{y} \\
\mathrm{PbTe} / \mathrm{SnTe}\end{array}$} & HWE & I' & {$[18,29,30]$} \\
\hline & $\begin{array}{l}\text { VPE } \\
\text { HWE }\end{array}$ & II & $\begin{array}{l}{[31]} \\
{[32,33]}\end{array}$ \\
\hline $\mathrm{PbTe} / \mathrm{Pb}_{1-x} \mathrm{Eu}_{x} \mathrm{Te}$ & HWE & I & {$[34-36]$} \\
\hline $\mathrm{PbTe} / \mathrm{Pb}_{1-x} \mathrm{Eu}_{x} \mathrm{Se}_{y} \mathrm{Te}_{1-y}$ & MBE & I & {$[37-39]$} \\
\hline $\mathrm{PbTe} / \mathrm{Pb}_{1-x} \mathrm{Ge}_{x} \mathrm{Te}$ & MBE & & {$[40]$} \\
\hline $\mathrm{PbTe} / \mathrm{CdTe}$ & ICB & & [41] \\
\hline $\mathrm{PbTe} / \mathrm{PbS}$ & VPE & & [9-11] \\
\hline $\mathrm{PbTe} / \mathrm{InSb}$ & LAE & & [12] \\
\hline $\mathrm{PbTe} / \mathrm{Bi}$ & VPE & $\begin{array}{l}\text { semiconductor/ } \\
\text { /semimetal }\end{array}$ & {$[42,43]$} \\
\hline $\mathrm{PbSe} / \mathrm{SnSe}$ & VPE & & [44] \\
\hline $\mathrm{PbS} / \mathrm{Pb}_{1-x} \mathrm{Eu}_{x} \mathrm{~S}$ & HWE & I & [45] \\
\hline \multirow[t]{2}{*}{$\mathrm{PbS} / \mathrm{PbSe}$} & VPE & I & {$[46]$} \\
\hline & $\begin{array}{l}\text { HWE } \\
\text { VPE }\end{array}$ & $\begin{array}{l}\mathrm{I} \\
\mathrm{I}\end{array}$ & $\begin{array}{l}{[47]} \\
{[48,49]}\end{array}$ \\
\hline $\mathrm{PbTe}$ & HWE & nipi & {$[7,8,16,50-52]$} \\
\hline $\mathrm{PbSe} / \mathrm{Pb}_{1-x} \mathrm{Eu}_{x} \mathrm{Se}$ & MBE & $\mathrm{I}^{\prime}$ & [53] \\
\hline $\begin{array}{l}\text { HWE - hot wall epitaxy, } \\
\text { VPE - vapour phase epit } \\
\text { MBE - molecular beam } \\
\text { ICB - ionized cluster bea } \\
\text { IAE - l }\end{array}$ & pitaxy, & & \\
\hline
\end{tabular}

\section{Band offsets and the types of IV-VI SL}

Knowledge of the band offsets between the constituents of SL and/or heterostructure is very important as they define the electronic and optical properties, 
type I $\quad \mathrm{Pb}_{1-x} \mathrm{Sn}_{\mathrm{x}} \mathrm{Te} \quad$ type I'

(a)

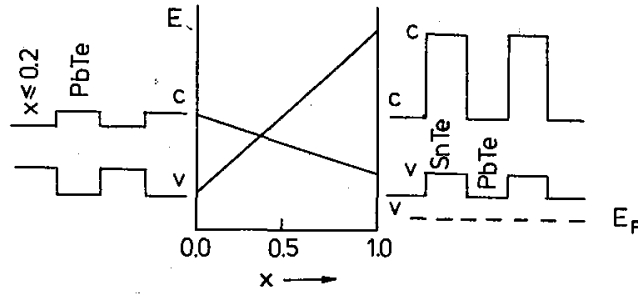

(b)

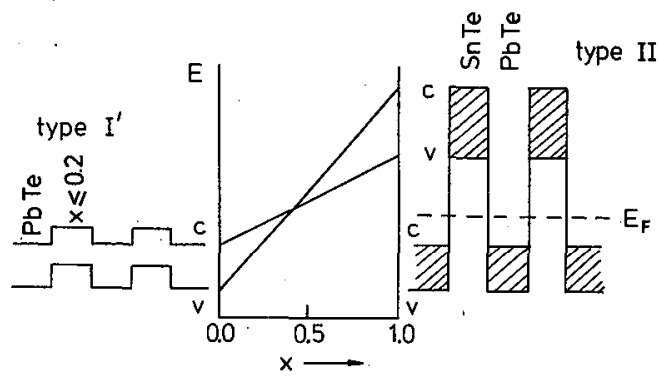

(c) type I

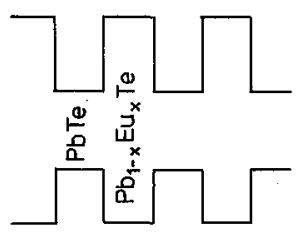

$\mathrm{PbTe} / \mathrm{Pb}_{1-x} \mathrm{Eu}_{x} \mathrm{Te}$

$\mathrm{PbTe} / \mathrm{Pb}_{1-x} \mathrm{Eu}_{x} \mathrm{Se}_{y} \mathrm{Te}_{1-y}$ $\mathrm{PbS} / \mathrm{PbSe}$ (d) type I'

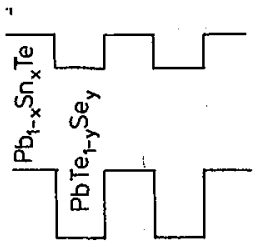

$\mathrm{Pb}_{1-x} \mathrm{Sn}_{x} \mathrm{Te}_{\mathrm{e}} / \mathrm{PbTe}_{1-y} \mathrm{Se}_{y}$ (e) type I

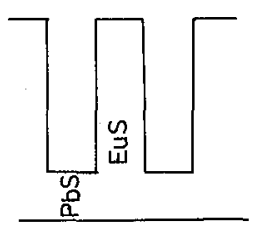

$\mathrm{PbS} / \mathrm{EuS}$

Fig. 3. Schematic band ordering in IV-VI SL and MQW: a) $\mathrm{PbTe} / \mathrm{Pb}_{1-x} \mathrm{Sn}_{x} \mathrm{Te}$ $(x \leq 0.20),[21-26]$, b) $\left.\mathrm{PbTe} / \mathrm{Pb}_{1-x} \mathrm{Sn}_{x} \mathrm{Te},[28,32,33], \mathrm{c}\right) \mathrm{PbTe} / \mathrm{Pb}_{1-x} \mathrm{Eu}_{x} \mathrm{Te}$ [34-36], $\mathrm{PbTe} / \mathrm{Pb}_{1-x} \mathrm{Eu}_{x} \mathrm{Se}_{y} \mathrm{Te}_{1-y}$ [37-39], $\mathrm{PbS} / \mathrm{PbSe}[46,47]$, d) $\mathrm{Pb}_{1-x} \mathrm{Sn}_{x} \mathrm{Te} / \mathrm{PbTe}_{-y} \mathrm{Se}_{y}$ $[18,29,30]$, e) $\mathrm{PbS} / \mathrm{EuS}[48,49]$. 
and so, the types of the optoelectronic devices. The band offsets result from the difference in the band structures of the constituents. They also depend on the orientation of the growth direction with respect to the crystallographic axes, the state of the surface, strains, band bending and other parameters. For a long time the band offsets were a subject of numerous discussions. Even in the case of GaAs/GaAlAs where the control of growth process and the state of interfaces is best, there exist discrepancies of about $0.1 \mathrm{eV}$ in the value of the conduction-band offset, e.g. depending on the experimental method of the measurment (see, e.g. [54]). For narrow-gap $\mathrm{PbTe} / \mathrm{Pb}_{1-x} \mathrm{Sn}_{x} \mathrm{Te}$ system the conduction-band offset is subject of a discussion which has not settled definitely by now. The conclusions of two leading groups dealing with these objects, obtained by the same method, are opposite (see Table 1). For other IV-VI systems, various types labelled I, II and type I' are possible (see Table I and Fig. 3).

Due to small values of the band gaps, especially in $\mathrm{PbTe} / \mathrm{Pb}_{1-x} \mathrm{Sn}_{x} \mathrm{Te}$ system $\left(E_{g}=0.19 \mathrm{eV}\right.$ and $0.08 \mathrm{eV}$ at $x=0$ and 0.20 respectively at $\left.T=0 \mathrm{~K}\right)$ and the smallness of the conduction band offset at $x \leq 0.20$, it is very important to take into account the strain-induced shift of the band edges. In the case of $\mathrm{PbTe} / \mathrm{Pb}_{1-x} \mathrm{Sn}_{x} \mathrm{Te}$ layers grown on (111) $\mathrm{BaF}_{2}$ substrates, the [111] valley oriented parallel to the growth direction is shifted downward in energy with respect to the remaining three oblique $\langle\overline{1} 11\rangle$ valleys $[21,24]$ (see Fig. 4). The strain-related
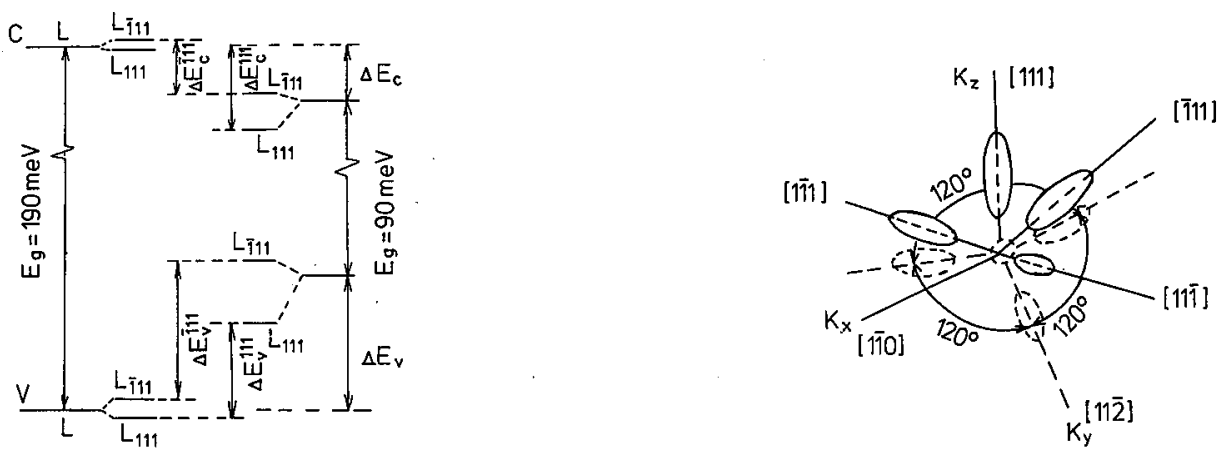

Fig. 4. The valence and conduction band edges of $\mathrm{PbTe}$ and $\mathrm{Pb}_{1-x} \mathrm{Sn}_{x} \mathrm{Te}$ in $\mathrm{PbTe} / \mathrm{Pb}_{1-x} \mathrm{Sn}_{x} \mathrm{Te} \mathrm{SL}$ on $\mathrm{BaF}_{2}$ substrate including strain induced energy shifts and splittings (after [24]).

Fig. 5. The orientation of tilted and "direct" valleys of equal energy surfaces in $\mathrm{Pb}_{1-x} \mathrm{Sn}_{x} \mathrm{Te}$.

change of the barrier height is rather significant and is of the order of the conduction band offset itself. The authors of Refs. [21-26] argued that $\mathrm{PbTe} / \mathrm{Pb}_{1-x} \mathrm{Sn}_{x} \mathrm{Te}$ $(x \leq 0.2) \mathrm{SL}$ are of type I (with the wells in narrower gap $\mathrm{Pb}_{1-x} \mathrm{Sn}_{x} \mathrm{Te}$ layers) basing themselves on different experimental results including: (a) magnetooptical experiments [55], giving the $g$-values of electrons in the wells equal to those in $\mathrm{Pb}_{1-x} \mathrm{Sn}_{x}$ Te films; (b) coherent Raman scattering [24]; (c) photoluminescence 
experiments $[25,26]$.

On the other hand in $[27,28]$ from the cyclotron resonance measurements it was shown that $\mathrm{PbTe} / \mathrm{Pb}_{0.8} \mathrm{Sn}_{0.2} \mathrm{Te} \mathrm{SL}$ are of type I', i.e. the the conduction band and valence band of $\mathrm{PbTe}$ are lower in energy than those in $\mathrm{Pb}_{0.8} \mathrm{Sn}_{0.2} \mathrm{Te}$. If the positions of the conduction and valence bands extrapolate linearly between the end-point materials $\mathrm{PbTe}$ and $\mathrm{SnTe}$, one obtains SL of type II when the valence band edge of SnTe is higher than the conduction band edge of PbTe (Fig. 3). In such case one can expect the electrons from valence band of SnTe to flow the conduction band of $\mathrm{PbTe}$, leading to a semimetallic structure. The magnetic field and temperature dependences of the Hall coefficient $R_{H}(H, T)$ of $\mathrm{PbTe} / \mathrm{SnTe}$ SL grown both on $\mathrm{BaF}_{2}$ [32] and $\mathrm{KCl}$ [33] substrates do show a large variations characteristic for the presence of two types of carriers. Moreover the investigations of $R_{H}(H)$ at $T=5 \mathrm{~K}$ show that $R_{H}(H)$ changes its sign from the negative to positive [33], strongly indicating that electrons with higher mobilities (in $\mathrm{PbTe}$ layers) and holes with lower mobilities (in SnTe layers) coexist in the structure. If the superlattices in question were of type $I$ then the linear extrapolation of the positions of band edges to $\mathrm{PbTe} / \mathrm{SnTe}$ case would lead to type I' SL for the latter pair of constituents (see Fig. 3). Then, such coexistence of carriers of different types is difficult to envision. If, on the other hand, $\mathrm{PbTe} / \mathrm{Pb}_{1-x} \mathrm{Sn}_{x} \mathrm{Te} \mathrm{SL}$ and/or MQW were of type $I$ then the realization of the lasers made only of $P_{1-x} \mathbf{S n}_{x} \mathrm{Te}$ constituents would be difficult. Untill now there are no communications in the literature on lasers made of $\mathrm{PbTe} / \mathrm{Pb}_{1-x} \mathrm{Sn}_{x} \mathrm{Te} \mathrm{MQW}$ or SL. Only the investigations of photoluminescence are reported $[25,26]$.

In all other cases of IV-VI SL, when the band gaps are wider than in $\mathrm{Pb}_{0.8} \mathrm{Sn}_{0.2} \mathrm{Te}$, there are no contradictions between different experiments and different groups. In majority of cases SL of type I are formed with the exception of $\mathrm{Pb}_{1-x} \mathrm{Sn}_{x} \mathrm{Te} / \mathrm{PbTe}_{1-y} \mathrm{Se}_{y}$ and $\mathrm{PbSe} / \mathrm{Pb}_{1-x} \mathrm{Eu}_{x} \mathrm{Se}$ where type I'. The examples of different types of SL are shown in Fig. 3.

\section{Preparation of IV-VI SL and MQW}

The growth of IV-VI SL or MQW was accomplished by HWE (hot wall epitaxy), MBE (molecular beam epitaxy) and VPE (vapour phase epitaxy) methods (see Table I). As most common substrates, the insulating cleaved (111) $\mathrm{BaF}_{2}$, (100) $\mathrm{KCl}$ or $\mathrm{NaCl}$ slices were used. (100) $\mathrm{PbTe}$ substrates, especially in the case of MQW lasers, were also employed. IV-VI semiconductors have rather large expansion coefficients $\alpha$ (see Table II), in particular, they differ considerably from the values of $\alpha$ of the insulating substrates. This introduces additional strains in IV-VI layers. To reduce this effect the buffer layers as a rule are grown.

The quality of the individual layers of IV-VI semiconductors grown by either MBE or HWE is rather similar. In both methods the IV-VI compounds are used as the source materials as a rule, rather than pure elements. The substrate temperatures are nearly the same $\left(T_{s} \approx 250-350^{\circ} \mathrm{C}\right)$ in both methods. In most of the cases this prevents interdiffusion of the components during the time of SL growth. Even for one of the most diffusive components, i.e. for $\mathrm{Sn}$, the interdiffusion effects are negligible in $\mathrm{PbTe} / \mathrm{Pb}_{1-x} \mathrm{Sn}_{x} \mathrm{Te} \mathrm{SL}$ at $T_{S} \leq 300^{\circ} \mathrm{C}[6,56]$. 
TABLE II

Lattice constants and expansion coefficients of some substrates with cubic symmetry used for growing IV-VI SL

\begin{tabular}{l|c|c|c}
\hline \hline Compound & $a_{0} \AA$ & $\Delta a_{0} / a_{0}, \%$ & $\alpha, 10^{-6} \mathrm{~cm} / \mathrm{K}$ \\
& $T=300 \mathrm{~K}$ & $\mathrm{~Pb}_{0.8} \mathrm{Sn}_{0.2} \mathrm{Te}$ & $T=300 \mathrm{~K}$ \\
\hline $\mathrm{Pb}_{0.8} \mathrm{Sn}_{0.2} \mathrm{Te}$ & 6.4321 & 0 & 20.0 \\
$\mathrm{PbTe}$ & 6.460 & -0.434 & 19.8 \\
$\mathrm{BaF}_{2}$ & 6.2001 & +3.61 & 18.0 \\
$\mathrm{KCl}$ & 6.2910 & +2.193 & 44.6 \\
$\mathrm{KBr}$ & 6.599 & -2.595 & 37.6 \\
$\mathrm{NaCl}$ & 5.65013 & +12.157 & 39.2
\end{tabular}

The growth rates are about $1-3 \mu \mathrm{m} / \mathrm{h}$ in both MBE and HWE methods, though in HWE they are normally a little faster. Both methods enable introduction of impurities in order to modify the composition of the layers and the concentrations of charge carriers in the layers. The great advantage of the MBE method is the possibility of in situ analysis of the quality of the layers. Nevertheless the experiments show that relatively simple HWE technique provides sufficient control over the growth processes of IV-VI semiconductors.

The electron or hole concentrations in the range of $\left(10^{17}-10^{19}\right) \mathrm{cm}^{-3}$ and the carrier mobility up to $\mu_{4 K} \approx 2 \times 10^{5} \mathrm{~cm}^{2} / \mathrm{V}$ 's may be achieved by the above methods in a controllable fashion opening the opportunity to construct IR optoelectronic devices according to a desirable design (see, e.g. $[17,29,34]$ ).

The electrical characteristics of IV-VI SL obtained by VPE method are not as good as required: the carrier concentrations are higher and the mobility is lower (see, e.g. $[10,11]$ ) than in the case of HWE or MBE techniques. Moreover there is almost no possibility to control these parameters in the process of growth.

It is rather difficult to discuss ionized cluster beam epitaxy (ICB) and laser assisted evaporation (LAE) methods in connection to IV-VI SL as there are only few papers devoted to them. The regimes used in [12] in the LAE method did not result in high quality IV-VI layers. However, it was shown in [57] that IV-VI layers of rather high quality with narrow diffraction peak half width $\left(\sim 40^{\prime}\right)$ can be obtained by this method if the substrates are kept at low temperature $\left(T_{S} \leq\right.$ $\left.150^{\circ} \mathrm{C}\right)$.

\section{Electronic structure of IV-VI SL}

The electronic structure of the SL is determined by the properties of the constituents. Since the electronic structure of various IV-VI semiconductors is rather similar, therefore IV-VI SL band structure in the vicinity of certain points in the Brillouin zone can easily be calculated within the framework of the envelope function approximation (EFA) (see, e.g. [21, 24, 58]). To calculate the SL electronic band structure the relative positions of the band edges of the constituents have to be known.

The values of dielectric constant $\varepsilon_{o}$ are high in narrow gap IV-VI semiconductors, especially at low temperatures $\left(\varepsilon_{o}=a \times 10^{5} /(b+T)\right.$, where $a$ and $b$ 
are constants [59]) the charge transfer across the interfaces does not give rise to a noticeable electrostatic potentials. The potential in question is of the order of several $\mathrm{meV}$ and, as a rule, is much smaller than the conduction or valence band offsets, although in some cases, at elevated temperatures, when $\varepsilon_{o}$-values are considerably reduced the band bending effect is not small. This explains why the laser action was observed in type I' SL [29]. Due to the large values of $\varepsilon_{0}$ the shape of the superlattice potential is almost rectangular which simplifies the Schrödinger equation. The electronic structure of IV-VI SL was calculated in many papers (see, e.g. [21, 22, 24, 58, 60,61] and references therein).

There are some differences between the electronic structures of IV-VI SL grown on (001) and (111) substrates. In the first case ( $\mathrm{PbTe}, \mathrm{KCl}$ being the substrates) all four valleys of IV-VI layers are oriented identically with respect to SL axis. In the second case $\left(\mathrm{BaF}_{2}\right.$ substrate) there are three tilted $\langle\overline{1} 11\rangle$ valleys with their main axes making an angle $\varphi$ with the SL axis, $\varphi=70.52^{\circ}$ (Fig. 5). In the last case the main axis of one [111] valley coincides with the SL axis. This leads to an energy splitting of the states in [111] and (i11) valleys (Fig. 6). To calculate the
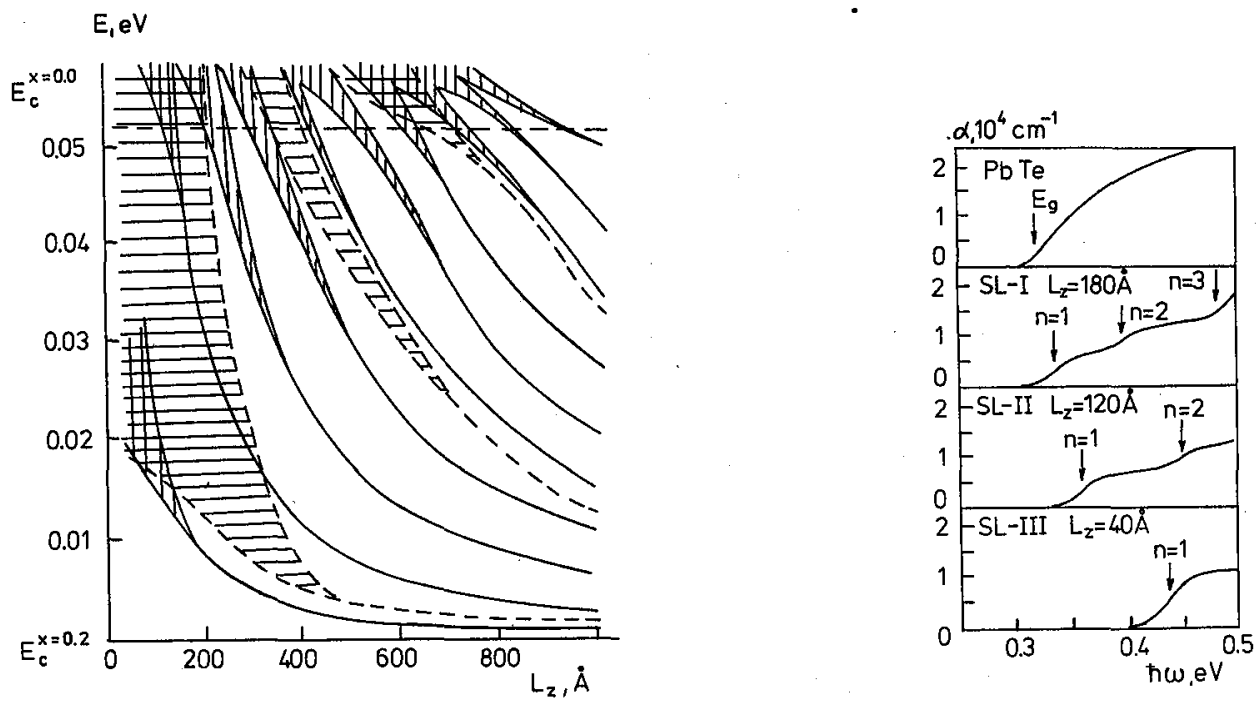

Fig. 6. Subband energies for electrons in the wells of $\mathrm{PbTe} / \mathrm{PbSnTe} \mathrm{MQW}$ as a function of the superlattice period, assuming $a=b=d_{0} / 2, \Delta E_{c}=\Delta E_{v}$ (strain not accounted for), at $T=150 \mathrm{~K}$. Solid lines show results for the "direct" valley, broken lines - for tilted valleys.

Fig. 7. Absorption coefficients $(\alpha)$ vs. photon energy of the PbTe film and $\mathrm{PbTe} / \mathrm{Pb}_{0.95} \mathrm{Eu}_{0.95} \mathrm{Te}$ SL [36].

energies of the minibands the following parameters of $\mathrm{Pb}_{1-x} \mathrm{Sn}_{x} \mathrm{Te}$ solid solutions together with their temperature dependencies were assumed (two-band effective mass approximation was employed) $E_{g}=0.186-0.531 x+4.5 \times 10^{-4} T^{2} /(T+20) \mathrm{eV}$; 
$m_{t}^{c, v}=\left(E_{g} / E_{\perp}\right) m_{o}$, where $E_{\perp}=2 m_{o} P_{\perp}^{2} / \hbar^{2}=6.8 \mathrm{eV} ; P_{\perp}=0.54 \mathrm{eVnm} ; K=$ $P_{1}^{2} / P_{\|}^{2}=m_{l} / m_{t}=10$. The dispersion relation in this case is:

$$
E_{c n, v m}(\vec{k})=-E_{g} / 2 \pm \frac{1}{2}\left[E_{g}^{2}+4 E_{c n, v m}^{2}+4\left(k_{y}^{2} P_{y}^{2}+k_{x}^{2} P_{x}^{2}+2 \alpha_{n m} k_{y}\right)\right]^{1 / 2} .
$$

Here $E_{c n}$ is the position of $n$-th miniband at $k_{\perp}=0, \alpha_{n}=E_{c n} P_{z y}^{2} P_{z}^{-1}$. For the [111] valley: $P_{z}=P_{\|} ; P_{x}, P_{y}=P_{\perp}, P_{z y}=0$. For the three $\langle\overline{1} 11\rangle$ valleys: $P_{x}=P_{\perp}$, $P_{y}^{2}=P_{\perp}^{2} \cos ^{2} \varphi+P_{\|}^{2} \sin ^{2} \varphi ; P_{z y}^{2}=\left(P_{\perp}^{2}-P_{\|}^{2}\right) \cos \varphi \sin \varphi ; P_{z}^{2}=P_{\|}^{2} \cos ^{2} \varphi+P_{\perp}^{2} \sin ^{2} \varphi$, where $\varphi$ is the angle between [111] and $\langle\overline{1} 11\rangle$ directions.

It is interesting to investigate the SL or MQW properties when there are only few fairly well separated minibands. As one can see from Fig. 6 for $\mathrm{PbTe} / \mathrm{PbSnTe}$ MQW on $\mathrm{BaF}_{2}$ substrate this is possible only for rather "thick" wells $(a \geq 200 \AA)$.

\section{Optical and electrical properties}

In IV-VI semiconductors with the extrema of the conduction and valence bands at the $L$ point of the Brillouin zone the direct dipole interband optical transitions occur. The contributions of each valley to the optical absorption coefficient is additive. In the case of SL on (100) substrates all valleys are equivalent. However, in the case of (111) substrates, due to energy inequality of the states in [111] and (111) valleys, there appears a dependence of the optical absorption on the polarization of light and as well as on the direction of its propagation with respect to the crystallographic axes [20,62]. The selection rule $\Delta n=0$ for the interband transitions takes place ( $n$ being the miniband index) although when the nonparabolicity of the bands is taken into account, the interband optical transitions with $\Delta n=1,3,5 \ldots$ are also allowed [61]. These latter transitions are much weaker than those with $\Delta n=0[61,62]$.

Typical dependencies of the absorption coefficient on the wavelength in IV-VI $\mathrm{SL}$ are shown in Fig. 7 for $\mathrm{PbTe} / \mathrm{Pb}_{0.95} \mathrm{Eu}_{0.05} \mathrm{Te} \mathrm{Sl}$ on a (100) substrate. The absorption edges for $n \rightarrow n$ transitions are clearly seen. The height of the steps is independent of the photon energy as it is expected from simple considerations involving joint density of states.

Weak field magnetoresistance (WFMR) measurements are frequently used to test the two-dimensional character of the conductivity of free carriers in IV-VI SL (see, e.g. [21, 34, 62]) as the angular dependences of WFMR are very pronounced in this case. Unfortunately in IV-VI structures there are also spurious contributions of the angular dependences of WFMR due to many valley character of the band structure and the strain related to the mismatch of the lattice constants of the substrates and SL layers. To illustrate this we show in Fig. 8 the angular dependences of WFMR for $\mathrm{Pb}_{0.8} \mathrm{Sn}_{0.2} \mathrm{Te}$ monocrystalline film and $\mathrm{PbTe} / \mathrm{Pb}_{0.8} \mathrm{Sn}_{0.2} \mathrm{Te}$ $\mathrm{MQW}$ on $\mathrm{BaF}_{2}$ substrate. The measurements were performed for configurations labeled B and C (see the inset), as proposed in [63]. The configuration B involves rotation of the magnetic field in the plane perpendicular to the current direction and configuration $\mathrm{C}$ involves rotation of the magnetic field from the transverse configuration to longitudinal configuration. 


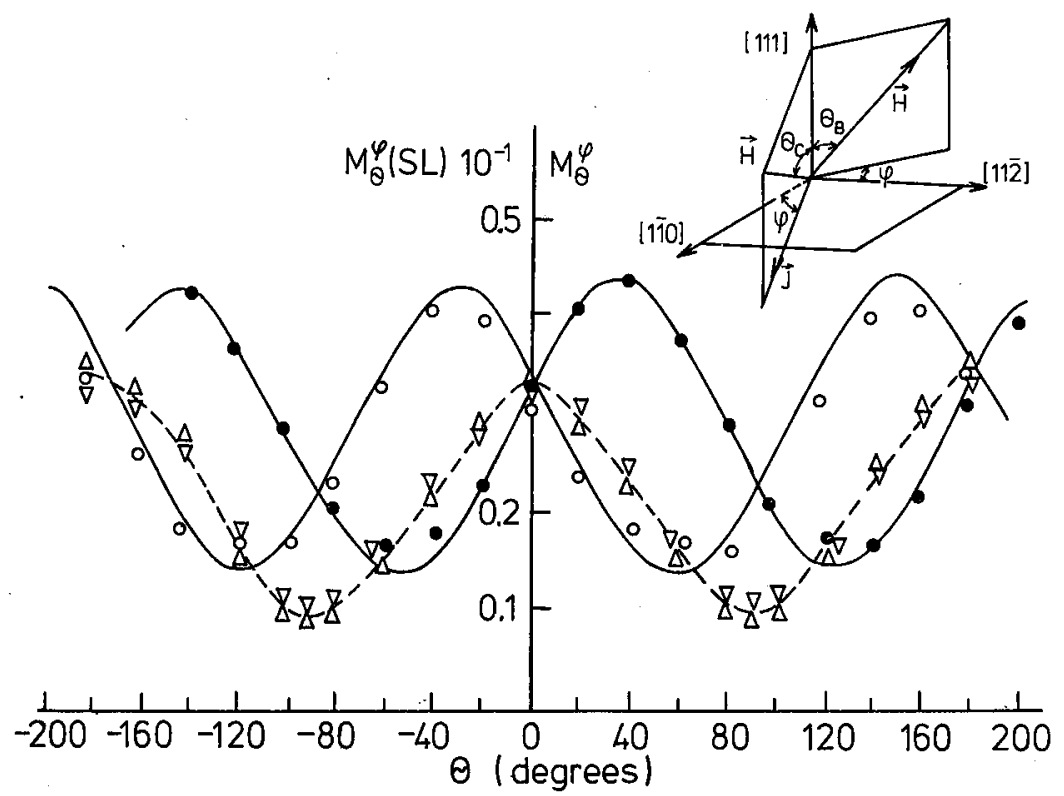

Fig. 8. The dependences of WFMR in p-type $\mathrm{PbTe} / \mathrm{Pb}_{0.8} \mathrm{Sn}_{0.2} \mathrm{Te} \mathrm{MQW}$ (triangles) and $\mathrm{Pb}_{0.8} \mathrm{Sn}_{0.2} \mathrm{Te}$ film (solid and empty circles). The solid curves show calculations for the film. The broken line represents the envelope curve of the experimental data for MQW. The parameters.of the MQW are $a=200 \AA, b=800 \AA, p_{77}=5.7 \times 10^{17} \mathrm{~cm}^{-3}, \mu_{77}=$ $5.1 \times 10^{3} \mathrm{~cm}^{2} / \mathrm{V} \mathrm{s}, d_{b u f f} \approx 1 \mu \mathrm{m}$. In the epitaxial film $d=3 \mu \mathrm{m}, p_{77}=2.95 \times 10^{17} \mathrm{~cm}^{-3}$, $\mu_{77}=1.42 \times 10^{4} \mathrm{~cm}^{2} / \mathrm{V} \mathrm{s}$.

To demonstrate the difference between WFMR angular dependences in epitaxial layers and MQW structure the value of $\varphi=45^{\circ}$ was chosen (by orienting the substrate properly) since in this case the angular dependences of WFMR in the film are almost opposite for the $B$ and $C$ configurations. For the MQW structure both configurations correspond to the same angular dependences, whose amplitude is one-order-of-magnitude higher than for quasi two dimensional system with an isotropic band stucture.

Another indication of $2 \mathrm{D}$ character of the conductivity in SL or MQW is provided by the angular dependence of the Shubnikov-de Haas oscillations (see, e.g. [64]). Moreover, in $\mathrm{PbTe} / \mathrm{Pb}_{0.78} \mathrm{Sn}_{0.22} \mathrm{Te} \mathrm{SL}$ on (111) $\mathrm{BaF}_{2}$ substrate an indication of the quantum Hall effect was observed for the first time in IV-VI systems [64].

IV-VI SL exhibit many other unusual features connected to the peculiarities of their band structure and other physical properties. For example the misfit dislocations in IV-VI dislocation SL form a square grid of edge misfit dislocations (MD) at the interface of IV-VI layers with a period $130 \AA$ (PbSe/PbS), $86 \AA(\mathrm{PbTe} / \mathrm{PbSe})$ and $52 \AA(\mathrm{PbTe} / \mathrm{PbS})[10,11]$ which is thought to be a reason of very high temper- 
atures of the superconducting phase transitions (as high as $T_{c}=5.5 \mathrm{~K}$ ) - in fact, the highest ever observed in semiconductors. Superconductivity in these systems is two-dimensional in nature. The average Hall concentration of electrons is about $N \approx(1-5) \times 10^{19} \mathrm{~cm}^{-3}$ and the carrier mobility in the normal phase does not exceed $(300-1200) \mathrm{cm}^{2} / \mathrm{V}$ s. Such dislocation SL seem to be close structural analogs of YBaCuO-type high temperature superconductors (HTSC) with the square MD grids imitating $\mathrm{CuO}_{2}$ planes in HTSC structure and being responsible for superconductivity.

\section{Final remarks}

This presentation cannot cover all known aspects of IV-VI SL physics. For example, electronic processes in nipi structures were not touched at all in the paper. They are discussed in detail in $[16,65,66]$. Concluding, we have tried to show that the new degree of freedom of design that exists in SL and MQW structures does indeed lead to new phenomena and new types of effective IR optoelectronic devices stemming from two dimensional character of the systems under consideration.

\section{References}

[1] L. Esaki, R. Tsu, IBM J. Res. Dev. 1970, p. 61.

[2] L.V. Keldysh, Fiz. Tverd. Tela 4, 2265 (1962).

[3] F. Capasso, in Semicond. and Semimetals, Vol.24, ed. R.K.Willordon, A.C. Beer, Academic Press, San Diego 1987, p.319.

[4] K. Ploog, G.H. Dohler, Adv. Phys. 32, 285 (1983).

[5] M.A. Herman, Semiconductor Superlattices, Akademie Verlag, Berlin 1986.

[6] H. Kinoshita, H. Fujiyasu, J. Appl. Phys. 51, 5845 (1980).

[7] H. Kinoshita, T. Sakashita, H. Fujiyasu, J. Appl. Phys. 52, 2869 (1981).

[8] H. Clemens, E.J. Fantner, G. Bauer, Rev. Sci. Instrum. 54, 685 (1983).

[9] L.S. Palatnik, A.I. Fedorenko, J. Cryst. Growth 52, 917 (1981).

[10] O.A. Mironov et al., JETP Lettr. 50, 300 (1989).

[11] O.A. Mironov et al., JETP Lettr. 48, 100 (1988).

[12] S.V. Gaponov, B.M. Luskin, N.N. Salashenko, Fiz. Tekh. Poluprovodn. 14, 1468 (1980).

[13] Yu.I. Ravich, B.A. Efimova, J.A. Smirnov, Semiconducting Lead Chalcogenides, Plenum Press, New York 1970.

[14] A. Rogalski, J. Piotrowski, Prog. Quantum. Electron. 12, 87 (1988).

[15] J.N. Walpole, A.R. Calawa, T.C. Harman, S.H. Groves, Appl. Phys. Lett. 28, 552 (1976).

[16] G. Bauer, W. Jantsch, Proc. SPIE-Int. Soc. Opt. Eng. 943, 107 (1988).

[17] D.L. Partin, Superlattices Microstruct. 1, 131 (1985).

[18] K. Shinohara et al., Appl. Phys. Lett. 47, 1184 (1985). 
[19]. M. Załużny, Acta Phys. Pol. A75, 19 (1989).

[20] J.V. Gumenjuk-Sichevskaya, F.F. Sizov, Ukrainian Fiz. Zhur. 34, 1811 (1989).

[21] M. Kriechbaum et al., Phys. Rev. B 30, 3394 (1984).

[22] P. Pichler et al., Superlattices Microstruct. 1, 1 (1985).

[23] H. Pascher et al., Surf. Sci. 170, 657 (1986).

[24] M. Kriechbaum, P. Kocevar, H. Pascher, G. Bauer, IEEE J. Quantum Electron. 24, 1727 (1988).

[25] M.V. Valeiko et al., Sov. Phys.-Semicond. 21, 34 (1987).

[26] M. Kriechbaum et al., Superlattices Microstruct. 5, 93 (1989).

[27] S. Shimomura et al., Superlattices Microstruct. 7, 5 (1990).

[28] K. Murase et al., Superlattices Microstruct. 1, 177 (1985).

[29] A. Ishida, H. Fujiyasu, H. Ebe, K. Shinohara, J. Appl. Phys. 59, 3023 (1986).

[30] A. Ishida, M. Aoki, H. Fujiyasu, J. Appl. Phys. 58, 797 (1985).

[31] M. A. Tamor et al., Superlattices Microstruct. 4, 493 (1988).

[32] A. Ishida, M. Aoki, H. Fujiyasu. J. Appl. Phys. 58, 1901 (1985).

[33] S. Takaoka et al., Solid State Commun. 58, 637 (1986).

[34] A. Ishida et al., Superlattices Microstruct. 2, 575 (1986).

[35] T. Nakamura, A. Ishida, H. Fujiyasu, Thin Solid Films 161, 149 (1988).

[36] A. Ishida, S. Matsuura, M. Mizuno, H. Fujiyasu, Appl. Phys. Lett. 51, 478 (1987).

[37] D.L. Partin, C.M. Trush, Appl: Phys. Lett. 45, 193 (1984).

[38] D.L. Partin, Appl. Phys. Lett. 45, 487 (1984).

[39] L.S. Kim et al., Phys. Rev. B 35, 2521 (1987).

[40] D.L. Partin, J. Vac. Sci. Technol. 21, 1 (1982).

[41] T. Takagi, H. Takaoka, Y. Kurijama, K. Matsubara, Thin Solid Films 126, 149 (1985).

[42] S.C. Shin, J.E. Hilliard, J.B. Ketterson, J.Vac. Sci. Technol. A 2, 296 (1984).

[43] A. Divenire et al., J. Cryst. Growth 70, 452 (1984).

[44] Z. Hiroi, N. Nakayama, Y. Bando, J. Appl. Phys. 61, 206 (1987).

[45] A. Ishida et al., Superlattices Microstruct. 6, 27 (1989).

[46] I.V. Kolesnikov et al., Fiz. Tekh. Poluprovodn. 23, 960 (1989).

[47] T.K. Chu, D. Agassi, A. Martinez, Appl. Phys. Lett. 50, 419 (1987).

[48] I.V. Kolesnikov et al., Zh. Exp. Teor. Fiz. 94, 239 (1988).

[49] I.V. Kolesnikov, A. Yu. Sipatov, Fiz. Tekh. Poluprovodn. 22, 954 (1989).

[50] P. Pichler et al., Superlattices Microstruct. 3, 225 (1987).

[51] W. Jantsch, G. Bauer, P. Pichler, H. Clemens, Appl. Phys. Lett. 47, 738 (1985). 
[52] G. Bauer, J. Oswald, W. Goltos, A.V. Nurmikko, J. Appl. Phys. 63, 2179 (1988).

[53] H. Heinrich et al., Supperlattices Microstruct. 5, 175 (1989).

[54] J.M. Langer, C. Delerue, M. Lawno, H. Heinrich, Phys. Rev. B 38, 7723 (1988).

[55] H. Pasher, G. Bauer, H. Clemens, Solid State Commun. 55, 765 (1985).

[56] E.J. Fantner, G. Bauer, Two Dimensional Systems, Heterostructures and Superlattices, eds. G. Bauer, F Kuchar, H. Heinrich, Springer Ser. Solid St. Sci. Vol.53, Berlin - Heidelberg 1984, p.207.

[57] S.V. Plyatsko, Yu.S. Gromovoj, F.F. Sizov, Infrared Phys. (to appear in 1990).

[58] M. Załużny, Phys. Rev. B 39, 12948 (1989).

[59] S. Nishi, H. Kawamura, K. Murase, Phys. Status Solidi B 97, 581 (1980).

[60] M. De Dios Leyva, J. Lopez Gondar, Phys.Status Solidi $B$ 138, 253 (1986).

[61] M. Załużny. Acta Phys. Pol. A75, 19 (1989).

[62] F.F. Sizov et al., Semicond. Sci. Technol. 5, 928 (1990).

[63] R.S. Allgaier, J.B. Restoff, B. Houston, Phys. Rev. B 19,6155 (1979).

[64] S. Takaoka et al., J. Phys. Soc. Jpn. 55, 2519 (1986).

[65] W. Jantsch, G. Bauer, Acta Phys. Pol. A73, 943 (1988).

[66] G.H. Dohler, Proc. SPIE-Int. Soc. Opt. Eng. 861, 21 (1987). 